\title{
"Radiation Recall Phenomenon" with Novel Cytotoxic Agents: An Emerging Trend in the Last Decade
}

\author{
Avik Mandal ${ }^{1}$ Pritanjali Singh ${ }^{1}$ Sarthak Tandon ${ }^{2}$ \\ ${ }^{1}$ Department of Radiation Oncology, All India Institute of Medical \\ Sciences, Patna, Bihar, India \\ ${ }^{2}$ Department of Radiation Oncology, Rajiv Gandhi Cancer Institute \\ and Research Centre, Delhi, India
}

Ind J Med Paediatr Oncol 2021;42:28-34.

\author{
Dharmendra Singh ${ }^{1}$
}

\begin{abstract}
Keywords

- immunotherapy

- radiation

- recall

- targeted therapy
\end{abstract}

\author{
Address for correspondence Avik Mandal, MBBS, DMRT, DNB \\ (Radiation Oncology), Department of Radiation Oncology, All India \\ Institute of Medical Sciences, Patna-Aurangabad Rd., Phulwari \\ Sharif, Patna 801 507, Bihar, India \\ (e-mail: dravikmandal@gmail.com).
}

\section{Introduction}

Radiation recall phenomenon (RRP) is an acute inflammatory reaction manifested to previously irradiated tissues after the administration of various pharmacologic compounds. With the completion of radiotherapy (RT), it may develop after an unpredictable, uncertain range of time interval of a few days to several years. D'Angio et al first reported the reactivation of "latent" radiation effects by intravenous administration of actinomycin D in 1959 at the Children's Medical Center in Boston. ${ }^{1}$ This response was described as the potentiation of X-ray effects that was sharply restricted to the previously treated area. Over the years, plenty of articles have implicated chemotherapeutic drugs as the most common causative agents of RRP, but various noncytotoxic drugs including antibiotics, ${ }^{2-4}$ lipid-lowering agents, ${ }^{5}$ and antitubercular drugs $^{6}$ have also been reported to cause it. Skin manifestations are the most common clinical features, but patients may present with visceral reactions including pneumonitis, hematuria, myopathy, and mucositis. In the last decade, various unconventional, novel agents were used increasingly in the field of oncology. In this narrative review article, the reported incidences of RRP in the past 10 years with various novel cytotoxic drugs including immunotherapies, unconventional chemotherapies, and molecular targeted agents with special emphasis on nivolumab and vemurafenib have been analyzed.
DOI https://doi.org/ $10.1055 / \mathrm{s}-0041-1729729$ ISSN 0971-5851
(C) 2021. Indian Society of Medical and Paediatric Oncology

This is an open access article published by Thieme under the terms of the Creative Commons Attribution-NonDerivative-NonCommercial-License, permitting copying and reproduction so long as the original work is given appropriate credit. Contents may not be used for commercial purposes, or adapted, remixed, transformed or built upon. (https://creativecommons.org/licenses/by-nc-nd/4.0/).

Thieme Medical and Scientific Publishers Pvt. Ltd. A-12, 2nd Floor, Sector 2, Noida-201301 UP, India 


\section{Pathophysiology of Radiation Recall Phenomenon}

This reaction is idiopathic, for which pathophysiology is not well established. Several hypotheses, that is, (1) vascular damage, (2) epithelial stem cell inadequacy, (3) epithelial stem cell sensitivity, and (4) drug hypersensitivity reaction, are proposed as the potential factors behind RRP.7 According to Hellman and Botnick ${ }^{8}$ and Seymour et $\mathrm{al}^{9}{ }^{9} \mathrm{RRP}$ is envisaged as the epithelial cell depletion triggered by the causative drugs, which is followed by an inadequate stem cell response. Local vascular permeability or proliferative changes in the previous radiation field may also affect the subsequent pharmacokinetics of drugs to induce RRP.

\section{Methods}

We retrieved all the prior publications since 2010 in any language including case reports, case series, abstracts, and poster presentations on RRP through a literature search of MEDLINE and PubMed using the keywords "radiation," "recall," "targeted therapy," and "immunotherapy," and references identified in retrieved articles were also used for further search of the literatures.

\section{Radiation Recall Phenomenon with Antiprogrammed Death-1/Programmed Death-Ligand 1}

Checkpoint antibody inhibitors, such as antiprogrammed death-(PD-1)/(PD-L1), are a novel class of cytotoxic drugs that suppresses tumor activity through modulation of immune cell-tumor cell interaction. Currently, the Food and Drug Administration (FDA) has approved different monoclonal antibodies targeting the PD-1/PD-L1 pathway, namely, nivolumab and pembrolizumab (PD-1 inhibitor), durvalumab, atezolizumab, and avelumab (PD-L1 inhibitor). These checkpoint inhibitors are reported to cause immune-related adverse events (irAEs) including cutaneous manifestations, ${ }^{10}$ but RRP caused by such agents is quite a novel phenomenon that we expect more to experience in the coming days with the increasing use of such promising drugs.

\section{Radiation Recall Dermatitis}

Two cases of radiation recall dermatitis (RRD) associated with the use of nivolumab were reported by Korman et $\mathrm{al}^{11}$ and Dhanushkodi et al. ${ }^{12} \mathrm{~A}$ young adult male with metastatic melanoma was treated with $9 \mathrm{~Gy}$ in three fractions of external beam RT to his left pelvis for the management of a pathologic fracture. He was started on nivolumab after 1 week, and he developed gradually increasing redness of the left flank and a geometric erythematous patch with fine scaling, conforming to the prior irradiation portals.

Another case of nivolumab-induced RRD was reported in a 34-year-old female with BRCA1-mutant, triple-negative metastatic breast cancer by Dhanushkodi et al. ${ }^{12}$ She was initially treated with conventional chemotherapies and received $30 \mathrm{~Gy}$ palliative chest wall radiation for the painful cutaneous nodules. Subsequently, she was started with nivolumab and after 10 days of the first dose of nivolumab, she developed fever and Grade 3 moist desquamation on her right chest wall that matched the previous RT fields, invoking the diagnosis of RRD.

The first-ever case of Stevens-Johnson syndrome (SJS) associated with RRD with administration of nivolumab was described in a 63-year-old male with pulmonary epidermoid carcinoma. ${ }^{13}$ He received three cycles of carboplatin-taxol, followed by thoracic RT (50 Gy on the mediastinum and left hilum, $60 \mathrm{~Gy}$ on the tumor, and $66 \mathrm{~Gy}$ on the surgical area). Later on, he received palliative radiation to the left knee and lumbar vertebrae and started with nivolumab. Five days after the second injection of nivolumab, erosive and painful lesions appeared inside the mouth. Extensive cutaneous lesions suggestive of SJS appeared 2 weeks after the second injection (-Table $\mathbf{1}$ ).

Table 1 Clinical detail of the reported cases of RRD with nivolumab in the past 10 years

\begin{tabular}{|c|c|c|c|c|}
\hline Study & Diagnosis & Dose and sites of RT & $\begin{array}{l}\text { Time interval between } \\
\text { completion of RT and } \\
\text { occurrence of RRD }\end{array}$ & Treatment \\
\hline Korman et al, $2017^{11}$ & Melanoma & $\begin{array}{l}9 \mathrm{~Gy} / \mathrm{3}^{\#} \text { left pelvis for patho- } \\
\text { logic fracture }\end{array}$ & 10 days & Topical corticosteroids \\
\hline Dhanushkodi et al, $2019^{12}$ & TNBC & 30 Gy to chest wall & 10 days & $\begin{array}{l}\text { Antibiotics, growth } \\
\text { factors, and intrave- } \\
\text { nous hydrocortisone }\end{array}$ \\
\hline $\begin{array}{l}\text { Rouyer et al, } 2018 \\
\text { (RDD with SJS) }^{13}\end{array}$ & $\begin{array}{l}\text { Pulmonary } \\
\text { epidermoid } \\
\text { carcinoma }\end{array}$ & $\begin{array}{l}50 \text { Gy mediastinum, left } \\
\text { hilum, } 60 \text { Gy on tumor, } 66 \\
\text { Gy on surgical area. } 30 \text { Gy } \\
\text { to left knee, and } 20 \text { Gy to } \\
\text { lumbar vertebrae over } \sim 11 \\
\text { months }\end{array}$ & $\begin{array}{l}15 \text { days after } R T+2 \text { cycles } \\
\text { of nivolumab }+5 \text { days after } \\
\text { the } 2^{\text {nd }} \text { cycle of nivolumab }\end{array}$ & NR \\
\hline van Seggelen et al, $2019^{21}$ & GBM & $\begin{array}{l}60 \mathrm{~Gy} / 30 \text { fractions along } \\
\text { with temozolomide }+ \text { stere- } \\
\text { otactic re-RT }\left(21 \mathrm{~Gy} / 3^{\#}\right)\end{array}$ & $\begin{array}{l}5 \text { days after re-RT }+50 \\
\text { days after last nivolumab }\end{array}$ & $\begin{array}{l}\text { Moderate-potency } \\
\text { topical corticosteroids } \\
\text { and prednisone }\end{array}$ \\
\hline
\end{tabular}

Abbreviations: GBM, glioblastoma multiforme; NR, not reported; RRD, radiation recall dermatitis; RT, radiotherapy; SJS, Stevens-Johnson syndrome; TNBC, triple-negative breast cancer.

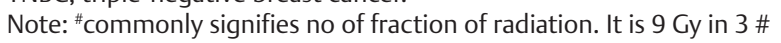




\section{Radiation Recall Pneumonitis}

Radiation recall pneumonitis (RRPn) describes a unique radiographic and clinical presentation of pneumonitis infiltrates in previously irradiated lung tissue after the use of triggering agents. The typical changes of RRPn, detected by contrast-enhanced computed tomography (CECT) scan, include ground-glass opacity, diffuse haziness, infiltrates, or consolidation in the irradiated lung that must match to the shape and size of the radiation portal. Ding et al ${ }^{14}$ described this rare phenomenon with 12 reported cases with conventional chemotherapy and found taxane to be the causative agent in the majority of recall pneumonitis.

The first report of RRPn by nivolumab was presented as a poster at ESMO Asia Congress 2016 by Miyoshi et al. ${ }^{15}$ A 44-year-old female with Stage IIIB nonsmall cell carcinoma of lung (NSCLC) underwent concurrent chemoradiotherapy (CRT) (60 Gy in 30 fractions). The patient received nivolumab as third-line chemotherapy for her disease progression. After three nivolumab dosing, and after 7 months of the cessation of RT, she developed clinical and radiological features of RRPn. Two similar cases of NSCLC who received $60 \mathrm{~Gy}$ of $\mathrm{RT}$ to thoracic region and subsequently developed RRPn with nivolumab had been reported by Shibaki et al. ${ }^{16}$ The radiological distribution of pneumonitis infiltrates corroborated the RT portals in both the cases.

Another case of RRPn with the use of nivolumab was reported in a 58-year-old female with dual malignancy. ${ }^{17}$ She was first treated with left mastectomy and adjuvant RT to a dose of $55 \mathrm{~Gy}$ for breast cancer and later, she received CRT to the upper part of the right hemithorax for adenocarcinoma lung. A new line of treatment with nivolumab was started as she developed metastases. Four weeks after the initiation of nivolumab, she developed clinical and imaging features of RRPn. One more patient with dual malignancy reported
RRPn-like pattern after multiple lines of conventional chemotherapy, nivolumab, and stereotactic body RT (SBRT) to thoracic lesion for Stage IV NSCLC. ${ }^{18}$ The patient subsequently developed chronic myelogenous leukemia and was started on imatinib. Over the next month, she developed radiological and clinical features of RRPn. Even though the immediate preceding agent was imatinib for this patient, the atypical nature of recall pneumonitis with imatinib warranted this case to be presented along with this review.

The incidence of RRPn induced by nivolumab had also been reported in a patient with renal cell carcinoma after palliative radiation (30 Gy in 10 fractions) to the ribs. ${ }^{19}$ Three days after the nivolumab administration, she presented with respiratory symptoms, and CECT scan image suggested recall pneumonitis.

RRPn was also reported in a 20-year-old female with relapsed Hodgkin lymphoma. ${ }^{20}$ She was started on nivolumab after the failure of conventional chemotherapy, total lymphoid irradiation (TLI), and stem cell transplant. She developed RRP after 16 months of TLI ( - Table 2 ).

\section{Radiation Recall Myelitis}

One case of radiation recall myelitis was reported in a 68-year-old male with metastatic NSCLC while on treatment with pembrolizumab. ${ }^{12}$ He developed motor deficit and sphincter dysfunction after $61 / 2$ months of radiation (30 Gy in 10 fractions to T12-L2 vertebra). The change in the signal intensity detected by magnetic resonance imaging (MRI) exactly corroborated the previously irradiated area. Because the radiation dose received by the thoracic spine was well below the recognized tolerance dose in this case, and other potential causes of myelopathy were ruled out, the most logical explanation was recall myelitis contributed by pembrolizumab.

Table 2 Clinical details of the reported cases of radiation recall pneumonitis with nivolumab in the past 10 years

\begin{tabular}{|c|c|c|c|c|}
\hline Study & Diagnosis & Dose and sites of RT & $\begin{array}{l}\text { Time interval } \\
\text { between completion } \\
\text { of RT and occurrence } \\
\text { of RRPn }\end{array}$ & Treatment \\
\hline Miyoshi et al, $2017^{15}$ & NSCLC & CRT (60Gy/30\#) & 7 months & NR \\
\hline \multirow[t]{2}{*}{ Shibaki et al, $2017^{16}$} & NSCLC & $60 \mathrm{~Gy} / 30^{\#}$ to thorax & $\begin{array}{l}2 \text { years gap }+4^{\text {th }} \text { cycle of } \\
\text { Nivolumab ( } 660 \text { days) }\end{array}$ & Oral prednisolone \\
\hline & NSCLC & $60 \mathrm{~Gy} / 30^{\#}$ to thorax & 664 days & Oral prednisolone \\
\hline Thoré et al, $2018^{17}$ & $\begin{array}{l}\text { Ca breast, adenocarci- } \\
\text { noma lung }\end{array}$ & $\begin{array}{l}\text { 55Gy for breast ca } \\
+ \text { CRT for ca lung + } \\
\text { palliative RT to bone }\end{array}$ & 7 months & NR \\
\hline Nakamura et al, $2019^{19}$ & Renal cell carcinoma & $30 \mathrm{~Gy} / 10^{\#}$ to ribs & 9 months & $\begin{array}{l}\text { Oral prednisolone for } 4 \\
\text { months }\end{array}$ \\
\hline Gupta et al, $2017^{20}$ & $\begin{array}{l}\text { Relapsed Hodgkin's } \\
\text { lymphoma }\end{array}$ & $\begin{array}{l}\text { Total lymphoid } \\
\text { irradiation }\end{array}$ & 16 months & Azithromycin + prednisolone \\
\hline Naiditch et al, $2019^{18}$ & $\mathrm{NSCLC}+\mathrm{CML}$ & $\begin{array}{l}\text { 54Gy/3\# SBRT to } \\
\text { thorax }\end{array}$ & 10 months & prednisolone \\
\hline
\end{tabular}

Abbreviations: CML, chronic myelogenous leukemia; CRT, concurrent chemoradiation; NR, not reported; NSCLC, nonsmall-cell lung carcinoma; RRPn, radiation recall pneumonitis; SBRT, stereotactic body radiation therapy. 


\section{Abscopal Recall Phenomenon}

RT that is a local modality of treatment can also stimulate systemic phenomena, and immune mechanisms triggered by RT are thought to be the underlying driving force behind such events. In 1953, R. H. Mole first coined the term "abscopal effects" of radiation to describe its effects "at a distance" (in Latin ab: away, scopal: target) from the irradiated volume. Similarly, the recall phenomenon of radiation can also be elicited at distant sites on very rare occasions.

A male patient with glioblastoma multiforme received 19 courses of nivolumab after completion of standard postoperative CRT (60 Gy in 30 fractions along with temozolomide) as the part of a trial study. ${ }^{21}$ One month after the last administration of nivolumab, he was treated with stereotactic re-irradiation (21 Gy/three fractions) for his recurrent disease. Five days after completing reradiation and 50 days after his last nivolumab administration, he developed a maculopapular rash in both armpits that later became generalized. This incidence can be explained by the "abscopal recall effect" of radiation triggered by nivolumab.

\section{Radiation Recall Phenomenon with BRAF Inhibitors}

BRAF inhibitors involve selective inhibition of the mutated BRAF V600E kinase, resulting in reduced signaling through the aberrant mitogen-activated protein kinase pathway, and FDA has approved three BRAF inhibitors, namely, vemurafenib, dabrafenib, and encorafenib, for the treatment of metastatic melanoma.

RT and vemurafenib are being increasingly used concurrently or sequentially, and vemurafenib is known to cause severe cutaneous reactions. Apart from the well-established cutaneous side effects and radiosensitization capacity of vemurafenib, there are several reported $\operatorname{cases}^{22-26}$ of RRP, with this BRAF inhibitor.

\section{Radiation Recall Dermatitis}

Three patients with metastatic, BRAF V600 E-positive melanoma, who developed RRP and RRD after the administration of vemurafenib, were reported by Forschner et al. ${ }^{27}$ Treatment with vemurafenib was started 3 to 4 weeks after the cessation of RT for all the three cases. Two patients in this case series received cumulative dose up to $50 \mathrm{~Gy}$ to the local site of the disease, which exposed pulmonary tissues as well. Features of RRP developed after 3 to 4 weeks of vemurafenib administration. Another patient who received $30 \mathrm{~Gy}$ whole-brain radiotherapy (WBRT) developed RRD in the form of multiple itchy vesicles and papules on the erythematous scalp, 2 weeks after starting vemurafenib. Similar two cases of RRD (cutis verticis gyrata) induced by vemurafenib after WBRT were reported by Harding et al. ${ }^{28}$

Interface dermatitis manifesting as RRD and a keratosis pilaris-like eruption were reported in a 37-year-old male who received adjuvant RT to his left neck ( $71 \mathrm{~Gy}$ in 38 fractions) after neck dissection for residual/recurrent melanoma. ${ }^{29} \mathrm{He}$ was put on vemurafenib 6 weeks after RT and a geometric area of dermatitis conforming to the previous radiation site developed after 2 weeks.
Localized multiple epidermal cysts were reported as recall phenomenon in a 43-year-old female with metastatic melanoma who received palliative RT to the exophytic tumor in the neck and started with vemurafenib. ${ }^{30}$ Three months after $\mathrm{RT}$, the patient was found to have multiple epidermal cysts in the previous irradiation field.

A patient with a diagnosis of BRAF-mutated, Stage IV adenocarcinoma lung received whole-femur palliative RT and, 8 weeks later, was initiated with oral dabrafenib and pazopanib. ${ }^{31}$ The patient developed RRD-like manifestations within few hours, corresponding to the previously irradiated area. Similar incidence with concomitant dabrafenib and trametinib has been reported in a patient with nodular melanoma in the parietal region of the scalp. ${ }^{32}$ After 2 years of interval, the patient received palliative RT to the right tibia, and RRD was found to develop subsequently.

\section{Radiation Recall Myelitis}

A 48-year-old female with metastatic melanoma received seven courses of nivolumab as first-line treatment and four courses of ipilimumab on disease progression..$^{33}$ Concomitant with ipilimumab, she received SBRT (56 Gy in eight fractions) to a large pulmonary metastasis. Dabrafenib and trametinib were initiated as third-line treatment, and the patient developed skin rashes on her back in the same volume matching to the previous RT field after 7 months. After 1 month, she developed back pain, and positron emission tomography (PET)-CT scan and MRI showed the evidence of radiation recall myelitis that was limited to her thoracic spine.

\section{Radiation Recall Hematuria and Cystitis}

Regorafenib is an oral multikinase inhibitor that targets angiogenic, stromal, and tyrosine kinase receptors. The occurrence of recall hematuria and cystitis after hyperthermia and regorafenib has been reported in a 66-year-old female with rectal adenocarcinoma. ${ }^{34}$ The patient was given regorafenib on disease progression after multiple lines of treatment, which includes CRT with FOLFIRI (5-fluorouracil, leucovorin, and irinotecan) and $50.4 \mathrm{~Gy}$ of radiation in 28 fractions $\sim 3$ years back. Regional deep hyperthermia along with regorafenib was induced weekly using a BSD-2000 device (BDS Medical Corporation, Salt Lake City, Utah, United States) with $300 \mathrm{~W} / 77.0 \mathrm{MHz}$ of power for a growing presacral tumor. The patient started gross hematuria 3 days after commencing hyperthermia. The triggering agent for radiation recall hemorrhagic cystitis for this case is most likely the combination of regorafenib and hyperthermia.

\section{Radiation Recall Phenomenon (Newer Agents) Eribulin}

Eribulin induces G2/M cell cycle arrest and apoptosis by binding with microtubules and impairing microtubule growth. The first-ever case of recall reaction to eribulin was recorded in a 61-year-old bilateral breast cancer patient who went through several lines of conventional chemotherapy, RT to chest wall, SBRT to the lung (48 Gy in four fractions) 
and liver lesions (60 Gy in three fractions), palliative RT to the pelvic region and ribs, and reradiation to supraclavicular lymph nodes. ${ }^{35}$ Two weeks after pelvic RT, and 3 months following supraclavicular RT, she was initiated with eribulin and after two cycles of eribulin, recall dermatitis developed over the left breast and supraclavicular area.

\section{Osimertinib}

A male patient with metastatic adenocarcinoma lung was treated initially with conventional chemotherapies and osimertinib that target the mutated epidermal growth factor receptor T790M. The patient received thoracic hypofractionated RT (55 Gy in 20 fractions) for disease progression and developed RRPn within 2 weeks of osimertinib resume. ${ }^{36}$ Time interval since the completion of RT and development of RRPn was $\sim 2$ months. The logic to explain this event as RRPn was that the patient initially tolerated osimertinib without pulmonary side effects and resumption of osimertinib after thoracic RT resulted in severe inflammatory pneumonitis. It was quickly responsive to corticosteroids, which was another strong argument in favor of RRPn. This report also reinforces the fact that potential pulmonary toxicities may happen when RT and tyrosine kinase inhibitors (TKIs) are used concurrently or sequentially. ${ }^{37}$

\section{Ixabepilone}

A 60-year-old female with carcinoma breast underwent postoperative RT to chest wall and multiple lines of chemotherapy for her metastatic disease..$^{38}$ The patient also received palliative RT (30.25 Gy to T6 through T12 and 27.50 Gy to T1 through T3 over 13 and 14 days) and 1 week later, she received ixabepilone that is a microtubule-stabilizing agent. Within a week, she developed odynophagia along with the manifestation of RRD on her chest and back, conforming to the radiation portals.

\section{Mogamulizumab}

It is a humanized monoclonal antibody targeting the CC chemokine receptor 4 that has shown efficacy against adult $\mathrm{T}$ cell leukemia/lymphoma (ATLL), peripheral T cell lymphoma, and cutaneous $\mathrm{T}$ cell lymphoma. A 65-year-old male with relapsed ATLL underwent RT (50.4 Gy) to the superior mediastinum..$^{39}$ Three weeks after the completion of RT, weekly mogamulizumab was started and after three courses, he developed a geometric area of RRD.

\section{Rare Report of Radiation Recall Myocarditis}

It was described in a 56-year-old male with adenocarcinoma of the lung who received stereotactic RT to the chest and cervical lymph nodes (total dose of $70 \mathrm{~Gy}$ ) and multiple lines of chemotherapy including cisplatin, docetaxel, bevacizumab, erlotinib, gemcitabine, and lastly sorafenib. ${ }^{40}$ Five months later, he developed new-onset chest pain, and PET-CT, echocardiography, and cardiac magnetic resonance demonstrated abnormal myocardial wall segments, which corresponded to the previously irradiated area. This is the first-ever reported case of radiation recall myocarditis and underpins the features of visceral recall phenomenon that often remain underdiagnosed due to idiosyncrasy and lack of clinical suspicion.

\section{Managing a Case of Radiation Recall Phenomenon}

\section{Diagnosis}

The diagnosis of recall phenomenon is usually established by clinical judgment and radiological evidence. Biopsies of the affected areas were taken by few reported cases that revealed nonspecific changes on hematoxylin and eosin staining. Such features on skin biopsies may include vacuolization in basal cells, psoriasiform epidermal hyperplasia, follicular hyperkeratosis, and apoptotic keratinocytes. For visceral recall phenomenon, clinical presentations, history of prior radiation, and radiological changes in that particular organ can sufficiently lead to the diagnosis after ruling out the common causes of inflammatory reactions.

\section{Treatment}

Majority of the recall phenomenon regress clinically over a period of few weeks to months with the measurements including (a) immediate withdrawal of the causative agents, (b) administration of topical steroids for recall dermatitis, and (c) administration of systemic steroids with tapered dose schedule for visceral manifestations. Rechallenge with the causative agents without any further reactions is noted with a substantial number of cases. Dose modification of the triggering agents after the recall phenomenon can be considered on the discretion of the treating oncologist.

\section{Discussion}

The field of oncology is a rapidly expanding horizon with the frequent advent of newer therapeutic molecules and in the last decade, we experienced tremendous development in immunotherapy and targeted agents. While many of those agents have been found to cause dermatological side effects as the part of irAEs spectrum, the synergistic effects of such agents with RT are more pronounced. Radiation recall events, which are distinct inflammatory reactions of previously irradiated tissues with apparently obscure pathophysiology, are reported in considerable number with such novel cytotoxic agents. In the process of literature search, RRP was found to occur with numerous unconventional agents including monoclonal antibodies, ${ }^{41,42}$ TKIs, ${ }^{43-47}$ hormonal agents, ${ }^{48}$ and antibiotics. ${ }^{49}$ Four cases of hemorrhagic cystitis as RRP with the use of cabazitaxel were reported in a case series. ${ }^{50}$ In certain scenarios, it is extremely difficult to distinguish between "true" radiation recall and radiation-induced dermatitis because many of those agents are well known to cause radiosensitization and localized cutaneous reactions after $\mathrm{RT}^{24}$ The reported time interval between radiation and administration of triggering agent is variable from days to years. If the event occurs within the excepted time limit of usual skin toxicity, the diagnosis of RRD and RT-induced acute dermatitis may present overlapping. On the other hand, if RT was given in the remote past, it may remain 
underdiagnosed due to lack of clinical suspicion. In our study, we focused mainly on the immunotherapies, BRAF inhibitors, and few more novel cytotoxic agents, used extensively in the last decade. These agents are found to cause recall dermatitis as well as myriad visceral recall phenomena, such as pneumonitis, myocarditis, cystitis, and myelitis. To diagnose such events, clinical judgment and radiological evidence may be considered sufficient for the majority of the cases. Only a few circumstances may require biopsy that reveals nonspecific inflammatory changes. Majority of the cases regress with conservative treatment with or without corticosteroids. Safe rechallenges of the triggering agents are reported in multiple cases as well. With the increasing use of unconventional, novel cytotoxic agents and targeted molecules, concurrent or sequentially with radiation, we expect more incidences of RRP in future that may present with either dermatological or visceral recall reactions.

\section{Financial Support and Sponsorship}

Nil.

\section{Conflicts of Interest}

There are no conflicts of interest.

\section{References}

1 D’Angio GJ, Farber S, Maddock CL. Potentiation of x-ray effects by actinomycin D. Radiology 1959;73:175-177

2 Kang SK. Images in clinical medicine. Radiation recall reaction after antimicrobial therapy. N Engl J Med 2006;354(6):622

3 Wernicke AG, Swistel AJ, Parashar B, Myskowski PL. Levofloxacin-induced radiation recall dermatitis: a case report and a review of the literature. Clin Breast Cancer 2010; 10(5):404-406

4 Garrahy I, Forman D. Nitrofurantoin-induced radiation recall dermatitis. J Community Hosp Intern Med Perspect 2019;9(3):279-281

5 Abadir R, Liebmann J. Radiation reaction recall following simvastatin therapy: a new observation. Clin Oncol (R Coll Radiol) 1995;7(5):325-326

6 Extermann M, Vogt N, Forni M, Dayer P. Radiation recall in a patient with breast cancer treated for tuberculosis. Eur J Clin Pharmacol 1995;48(1):77-78

7 Camidge R, Price A. Characterizing the phenomenon of radiation recall dermatitis. Radiother Oncol 2001;59(3):237-245

8 Hellman S, Botnick LE. Stem cell depletion: an explanation of the late effects of cytotoxins. Int J Radiat Oncol Biol Phys 1977;2(1-2):181-184

9 Seymour CB, Mothersill C, Alper T. High yields of lethal mutations in somatic mammalian cells that survive ionizing radiation. Int J Radiat Biol Relat Stud Phys Chem Med 1986;50(1):167-179

10 Sibaud V. Dermatologic reactions to immune checkpoint inhibitors: skin toxicities and immunotherapy. Am J Clin Dermatol 2018;19(3):345-361

11 Korman AM, Tyler KH, Kaffenberger BH. Radiation recall dermatitis associated with nivolumab for metastatic malignant melanoma. Int J Dermatol 2017;56(4):e75-e77

12 Dhanushkodi M, Iyer $\mathrm{P}$, Ananthi B, Krishnamurthy A. Nivolumab-induced radiation recall dermatitis (RRD) Indian J Gynecol Oncol 2019;17:1-2
13 Rouyer L, Bursztejn AC, Charbit L, Schmutz JL, Moawad S. Stevens-Johnson syndrome associated with radiation recall dermatitis in a patient treated with nivolumab. Eur J Dermatol 2018;28(3):380-381

14 Ding X, Ji W, Li J, Zhang X, Wang L. Radiation recall pneumonitis induced by chemotherapy after thoracic radiotherapy for lung cancer. Radiat Oncol 2011;6:24

15 A First Report of Nivolumab induced Radiation Recall Pneumonitis | OncologyPRO. Available from: https:// oncologypro. Esmo. org/meeting-resources/esmo-asia-2016-congress/ A-First-Report-of-Nivolumab-induced-Radiation-RecallPneumonitis. Accessed April 12, 2021

16 Shibaki R, Akamatsu H, Fujimoto M, Koh Y, Yamamoto N. Nivolumab induced radiation recall pneumonitis after two years of radiotherapy. Ann Oncol 2017;28(6):1404-1405

17 Thoré P, Godbert B, Petit I, Chaouat A. Radiation recall pneumonitis in a patient treated by nivolumab for non-small cell lung cancer, no relapse with rechallenge. J Med Oncol 2018;3:12

18 Naiditch H, Brandsdorfer C, Possick JD. Imatinib-Induced Pneumonitis Associated with "Radiation-Recall" Pattern in a Patient with Chronic Myelogenous Leukemia and Prior Chemotherapy, Radiation Therapy, and Immunotherapy for Non-Small Cell Lung Cancer. In: American Thoracic Society International Conference Meetings Abstracts. American Thoracic Society; 2019. p. A1472-2

19 Nakamura K, Okubo K, Takahashi T, Mitsumori K, Ishigaki T, Ohnishi H. Radiation recall pneumonitis induced by nivolumab in a patient with renal cell carcinoma. IJU Case Rep 2018;2(1):30-33

20 Gupta K, Lee R, Sloan M, Diffuse lung disease session title: Drug-induced lung disease session type: Affiliate Case report poster presented nivolumab-induced radiation recall pneumonitis. 2017:152(4);A463. Available at https://journal.chestnet. org/article/S0012-3692(17)32008-1/fulltext. Accessed April 21

21 van Seggelen WO, De Vos FY, Röckmann H, van Dijk MR, Verhoeff JJ. Occurrence of an abscopal radiation recall phenomenon in a glioblastoma patient treated with nivolumab and re-irradiation. Case Rep Oncol 2019;12(3):896-900

22 Wang CM, Fleming KF, Hsu S. A case of vemurafenib-induced keratosis pilaris-like eruption. Dermatol Online J 2012;18(4):7

23 Anker CJ, Ribas A, Grossmann AH, et al. Severe liver and skin toxicity after radiation and vemurafenib in metastatic melanoma. J Clin Oncol 2013;31(17):e283-e287

24 Boussemart L, Boivin C, Claveau J, et al. Vemurafenib and radiosensitization. JAMA Dermatol 2013;149(7):855-857

25 Ducassou A, David I, Delannes M, Chevreau C, Sibaud V. Radiosensibilisation induite par le vémurafénib. Cancer Radiother 2013;17(4):304-307

26 Conen K, Mosna-Firlejczyk K, Rochlitz C, et al. Vemurafenibinduced radiation recall dermatitis: case report and review of the literature. Dermatology 2015;230(1):1-4

27 Forschner A, Zips D, Schraml C, et al. Radiation recall dermatitis and radiation pneumonitis during treatment with vemurafenib. Melanoma Res 2014;24(5):512-516

28 Harding JJ, Barker CA, Carvajal RD, Wolchok JD, Chapman PB, Lacouture ME. Cutis verticis gyrata in association with vemurafenib and whole-brain radiotherapy. J Clin Oncol 2014;32(14):e54-e56

29 Braunstein I, Gangadhar TC, Elenitsas R, Chu EY. Vemurafenibinduced interface dermatitis manifesting as radiation-recall and a keratosis pilaris-like eruption. J Cutan Pathol 2014;41(6):539-543

30 Houriet C, Klass ND, Beltraminelli H, Borradori L, Oberholzer PA. Localized epidermal cysts as a radiation recall phenomenon in a melanoma patient treated with radiotherapy 
and the BRAF inhibitor vemurafenib. Case Rep Dermatol 2014;6(3):213-217

31 Haraldsdottir S, Bertino E, Haglund K, Kaffenberger B, Shah MH. Radiation recall dermatitis with concomitant dabrafenib and pazopanib therapy. JAMA Dermatol 2016;152(5):587-589

32 Yilmaz M, Celik U, Hascicek S. Radiation recall dermatitis with dabrafenib and trametinib: a case report. World J Clin Cases 2020;8(3):522-526

33 Ejlsmark MW, Kristiansen C, Eriksen JG, Hansen O, Bastholt L. Recall radiation myelitis after stereotactic radiation and dabrafenib in metastatic melanoma. Acta Oncol 2017;56(1):109-110

34 Zheng YM, Chow JM, Chang CL, Chao LH, Whang-Peng J, Lai GM. Hematuria induced by combination regorafenib and hyperthermia - a radiation recall effect. Int J Hyperthermia 2019;36(1):1186-1189

35 Tran WT, Ibáñez C, Pinto MP, Sanchez C, Czarnota GJ, Merino T. Eribulin-induced radiation recall dermatitis: a case report and brief review of the literature. Ecancermedicalscience 2020;14:1006

36 Sanchis-Borja M, Parrot A, Sroussi D, Rivin Del Campo E, Fallet V, Cadranel J. Dramatic radiation recall pneumonitis induced by osimertinib after palliative thoracic radiotherapy for lung cancer. J Thorac Oncol 2019;14(10):e224-e226

37 Togashi Y, Masago K, Mishima M, Fukudo M, Inui K. A case of radiation recall pneumonitis induced by erlotinib, which can be related to high plasma concentration. J Thorac Oncol 2010;5(6):924-925

38 Burris HA III, Hurtig J. Radiation recall with anticancer agents. Oncologist 2010;15(11):1227-1237

39 Sasaki K, Iinuma S, Fujii M, et al. Radiation recall dermatitis induced by mogamulizumab. J Eur Acad Dermatol Venereol 2020;34(2):e107-e108
40 Masri SC, Misselt AJ, Dudek A, Konety SH. Radiation recall reaction causing cardiotoxicity. J Cardiovasc Magn Reson 2014;16:25

41 Moon D, Koo JS, Suh CO, Yoon CY, Bae J, Lee S. Radiation recall dermatitis induced by trastuzumab. Breast Cancer 2016;23(1):159-163

42 Lee HE, Jeong NJ, Lee Y, et al. Radiation recall dermatitis and pneumonitis induced by trastuzumab (Herceptin ${ }^{\circledR}$ ) Int J Dermatol 2014;53(3):e159-e160

43 Chung C, Dawson LA, Joshua AM, Brade AM. Radiation recall dermatitis triggered by multi-targeted tyrosine kinase inhibitors: sunitinib and sorafenib. Anticancer Drugs 2010;21(2):206-209

44 Azad A, Maddison C, Stewart J. Radiation recall dermatitis induced by pazopanib. Onkologie 2013;36(11):674-676

45 Levy A, Hollebecque A, Bourgier C, et al. Targeted therapy-induced radiation recall. Eur J Cancer 2013;49(7):1662-1668

46 Clark D, Gauchan D, Ramaekers R, Norvell M, Copur MS. Radiation recall pneumonitis during systemic treatment with everolimus. Oncol Res 2014;22(5-6):321-324

47 Visy A, Bachelot T, Racadot S. Radiation recall syndrome in a patient with breast cancer, after introduction of everolimus. Cancer Radiother 2019;23(5):423-425

48 Ubukata M, Kamio T, Ohchi T, Noguchi E, Tsukada H, Kameoka S. Radiation recall dermatitis occurring 6 years and 4 months after breast-conserving surgery: a case report. Oncol Lett 2016;11(5):3071-3074

49 Rafiroiu S, Vassil A, Valente SA. Quinolone-induced radiation recall dermatitis in breast cancer patient. Breast J 2020;26(7):1407-1408

50 Grellety T, Houédé N, Hoepffner JL, et al. Hemorrhagic cystitis in patients treated with cabazitaxel: a radiation recall syndrome? Ann Oncol 2014;25(6):1248-1249 